\title{
12-hydroxyeicosatetraenoic acid is associated with variability in aspirin-induced platelet inhibition
}

Benjamin H Maskrey ${ }^{1}$, Gordon F Rushworth², Matthew H Law ${ }^{1}$, Andrew T Treweeke ${ }^{1}$, Jun Wei ${ }^{1}$, Stephen J Leslie ${ }^{3}$, Ian L Megson ${ }^{1}$ and Phillip D Whitfield ${ }^{1 *}$

\begin{abstract}
Background: Aspirin is one of the most widely used non-steroidal anti-inflammatory drugs (NSAIDs). It is also a commonly used anti-platelet drug, which inhibits the formation of the platelet activator, thromboxane $\mathrm{A}_{2}\left(\mathrm{TxA}_{2}\right)$ via inhibition of cyclooxygenase-1 (COX-1). However, the presence of a patient subset that fails to respond to aspirin despite reduced $\mathrm{TXA}_{2}$ concentrations suggests that the effect of aspirin might be more complex than exclusive COX-1 inhibition.

Methods: In this study we evaluated the impact of in vivo oral administration of a standard anti-platelet dose (75 mg) of aspirin in healthy volunteers on the acute impact of in vitro collagen-mediated platelet aggregation and generation of platelet-derived $\mathrm{TXA}_{2}$ and the 12-lipoxygenase (LOX) metabolite 12-hydroxyeicosatetraenoic acid (12-HETE). The eicosanoids were quantified using liquid chromatography-tandem mass spectrometry (LC-MS/MS).

Results: Low-dose aspirin administration not only inhibited $\mathrm{TXA}_{2}$ generation but also decreased the production of 12-HETE. Furthermore, a significant correlation was observed between the levels of 12-HETE and collagen-induced platelet aggregation. Pre-treatment of platelets with the 12-LOX inhibitor, baicalein, prior to activation attenuated platelet aggregation.

Conclusions: These findings support a role for 12-HETE as a pro-aggregatory eicosanoid in platelet function and suggest a role for 12-HETE in variable sensitivity to aspirin. The study also highlights a potentially important mechanism by which aspirin impacts upon eicosanoid generation.
\end{abstract}

Keywords: Aspirin, Platelet, 12-HETE, Thromboxane, Eicosanoids, Anti-inflammatory

\section{Background}

Aspirin (acetylsalicylic acid) is one of the most widely used non-steroidal anti-inflammatory drugs (NSAIDs). In addition to possessing anti-inflammatory properties at high doses (1 g/day) [1] aspirin also demonstrates effective cardioprotective properties at lower doses (75-150 mg/day) [2]. The proposed mechanism by which aspirin acts is by irreversible inhibition of cyclooxygenase 1 (COX-1) [3]. Enzyme acetylation effectively blocks formation of the prostaglandin intermediates, $\mathrm{PGG}_{2}$ and $\mathrm{PGH}_{2}$ and their resultant downstream bioactive products [4]. The cardioprotective anti-

\footnotetext{
* Correspondence: phil.whitfield@uhi.ac.uk

${ }^{1}$ Department of Diabetes and Cardiovascular Science, University of the Highlands and Islands, Old Perth Road, Inverness IV2 3JH, UK Full list of author information is available at the end of the article
}

platelet property of low-dose aspirin is attributed to irreversible inhibition of synthesis of a pro-thrombotic COX-1derived eicosanoid, thromboxane $\mathrm{A}_{2}\left(\mathrm{TxA}_{2}\right)$ [5], which is derived from the polyunsaturated fatty acid substrate, arachidonic acid (AA).

Platelet specificity of aspirin action is believed to stem from the fact that platelets lack nuclear DNA, rendering them incapable of de novo biosynthesis of COX-1, ensuring that inhibition of COX-1 prevails for the lifespan of the platelet. There is an increasing awareness of clinical variability in anti-thrombotic response to aspirin, with a significant proportion of patients failing to respond, a phenomenon referred to as 'aspirin resistance' [6]. Estimates of aspirin resistance vary widely (5-45\%) [7], but resistant patients are known to display an increased risk of 
cardiovascular morbidity [8]. There is, however, a lack of association between aspirin-mediated inhibition of platelet function and inhibition of $\mathrm{TxA}_{2}$ synthesis. This apparent anomaly has been attributed to a perceived lack of reliability of platelet function assays, on the assumption that reduced inhibition of $\mathrm{TxA}_{2}$ is the main contributory factor in aspirin resistance [9].

Whilst $\mathrm{TxA}_{2}$ is undoubtedly an important mediator in the platelet activation process, to date little attention has been paid to other eicosanoids generated by platelets. 12-hydroxyeicosatetraenoic acid (12-HETE), formed by the enzyme 12-lipoxygenase (12-LOX), is another major platelet-derived eicosanoid, although its role in platelet function remains unclear. Reports have suggested 12HETE to have both anti- and pro-thrombotic properties [10-12]. It has also been suggested to play a role in promoting expression of platelet CD62 (P-selectin) [13] and is elevated in patients with essential hypertension [14]. The increasing recognition of the potential importance of 12-HETE in platelet function has led to a recent resurgence of research in the area, which has resulted in the development of novel 12-LOX inhibitors to help elucidate the function of platelet 12-HETE and its potential as a novel anti-platelet target $[15,16]$. The impact of aspirin on 12-HETE is not yet fully understood. In this study we have investigated the association of $\mathrm{TxA}_{2}$ (measured as its degradation product $\mathrm{TxB}_{2}$ ) and 12-HETE levels, with low-dose aspirin-mediated inhibition of platelet aggregation in healthy volunteers.

\section{Methods}

\section{Subjects}

Healthy volunteers $(n=19)$ were recruited through the Highland Clinical Research Facility. Subjects were free from all non-steroidal anti-inflammatory drugs (NSAIDs) two weeks prior to blood collection. A $5 \mathrm{ml}$ blood sample was drawn into trisodium citrate tubes (Monovette ${ }^{\ominus}$, Sarstedt, Leicester, UK) from the antecubital fossa (21G) before and $1.5 \mathrm{~h}$ after oral dosing with aspirin (Actavis, Barnstaple, UK; $75 \mathrm{mg}$ ). For inhibitor studies, a further three healthy volunteers were recruited and blood drawn as above. The study was approved by the North of Scotland Research Ethics Committee and the University of the Highlands and Islands Ethics Committee, and was in accordance with the Declaration of Helsinki and its amendments, with all volunteers providing written informed consent.

\section{Measurement of platelet aggregation and inhibitor studies}

Platelet aggregation was measured in response to collagen in platelet-rich plasma (PRP) derived from the freshly drawn blood samples immediately before and $1.5 \mathrm{~h}$ after aspirin administration. Briefly, fresh blood was centrifuged ( $120 \mathrm{~g}$ for $10 \mathrm{~min}$ at room temperature), PRP aspirated and the platelet count obtained on an AcT Diff 8 Blood Counter (Beckman Coulter, High Wycombe, UK). The remaining blood sample was centrifuged $(1000 \mathrm{~g}$ for $10 \mathrm{~min}$ at room temperature) and the platelet poor plasma (PPP) aspirated for use as a reference and to dilute the PRP to a standard platelet count $\left(150 \times 10^{6} / \mathrm{ml}\right)$. Standardized PRP samples were aliquoted $(500 \mu \mathrm{l})$ into glass cuvettes with a micro-stirrer and pre-warmed to $37^{\circ} \mathrm{C}$ prior to addition of type I collagen (Labmedics, Manchester, UK; $2.5 \mu \mathrm{g} / \mathrm{ml}$ ). Aggregation was recorded for 6 min using a Chrono-Log 700 platelet aggregometer (Chrono-Log, Haverford, PA, USA), at which point the activated platelets were immediately stored at $-80^{\circ} \mathrm{C}$ for subsequent eicosanoid analysis. Aggregation was quantified as the area under the curve (AUC) over the $6 \mathrm{~min}$ period post-activation. To determine the effect of 12-LOX inhibition on platelet aggregation, platelets were pre-incubated for $10 \mathrm{~min}$ with either $100 \mu \mathrm{M}$ baicalein (Tocris Bioscience, Bristol, UK) or DMSO as a vehicle control at a final concentration of $0.5 \%(\mathrm{v} / \mathrm{v})$ prior to addition of $1.25 \mu \mathrm{g} / \mathrm{ml}$ collagen; aggregation was measured as described above and samples retained at the end of the aggregation assay for subsequent eicosanoid measurements.

\section{LC-MS/MS analysis of platelet eicosanoids}

Eicosanoids were isolated from the PRP following collagen activation using solid-phase extraction (SPE) chromatography (C18 $500 \mathrm{mg}, 6 \mathrm{ml}$, Waters, Manchester, UK). $1 \mathrm{ml}$ ice-cold methanol containing $1 \mathrm{ng}$ of the deuterated internal standards, $\mathrm{PGE}_{2}-\mathrm{d}_{4}$ and15-HETE- $\mathrm{d}_{8}$ (Cayman Chemical, Ann Arbor, MI, USA), was added to $0.5 \mathrm{ml}$ PRP to precipitate proteins, and following centrifugation $(600 \mathrm{~g}$ for $10 \mathrm{~min}$ at $4^{\circ} \mathrm{C}$ ), the supernatant was removed, diluted

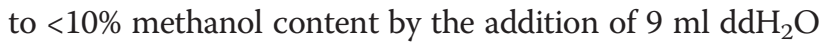
and acidified to $\mathrm{pH} 3.5$ with $1 \mathrm{M} \mathrm{HCl}$. Acidified samples were immediately added to SPE cartridges, which had been conditioned with $2 \times 6 \mathrm{ml}$ methanol, followed by $2 \times$

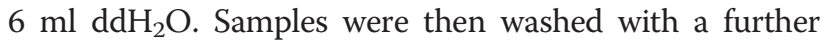
$6 \mathrm{ml} \mathrm{ddH_{2 }} \mathrm{O}$ and $2 \times 5 \mathrm{ml}$ hexane before elution of the eicosanoids with $2 \times 3 \mathrm{ml}$ ethyl acetate. The ethyl acetate fraction was dried under vacuum and then resuspended in $100 \mu \mathrm{l} 50: 50(\mathrm{v} / \mathrm{v})$ solvent A:solvent B where solvent A consisted of $\mathrm{H}_{2} \mathrm{O}$ :methanol 90:10 (v/v) containing 0.1\% (v/v) acetic acid and solvent B consisted of methanol containing $0.1 \%(\mathrm{v} / \mathrm{v})$ acetic acid. All solvents were LC-MS grade (Fisher Scientific, Loughborough, UK).

Eicosanoids were separated on a Kinetex $1.7 \mu \mathrm{m}$ XBC18 column $(100 \times 2.1 \mathrm{~mm})$ (Phenomenex, Macclesfield, UK) column using a Thermo Accela 1250 UHPLC system (Thermo Scientific, Hemel Hempstead, UK). The gradient was $45-100 \%$ solvent B over $18 \mathrm{~min}$ at a flow rate of $400 \mu \mathrm{l} / \mathrm{min}$. The LC effluent was directed into the source of a Thermo TSQ Quantum Ultra triple quadrupole mass spectrometer. The instrument was operated in negative 
ion mode and data were acquired using the selected reaction monitoring (SRM) mode. Eicosanoids were identified on the basis of their characteristic ion pairs $\left(\mathrm{TxB}_{2}, 369 /\right.$ 169; $\mathrm{PGE}_{2}-\mathrm{d}_{4}$, 355/193; $\mathrm{PGE}_{2}, 351 / 189$; 12-HETE, 319/ 179; 15-HETE- $\left.\mathrm{d}_{8}, 327 / 226\right)$ and matching retention time with authentic standards. Data were acquired and analysed using Thermo LCquan v2.6 software. Concentrations of $\mathrm{TxB}_{2}$ and 12-HETE were determined by comparison to a calibration curve run in parallel for each compound and adjusted for recovery by reference to amounts of the $\mathrm{PGE}_{2}-\mathrm{d}_{4}$ and 15-HETE- $\mathrm{d}_{8}$ internal standards respectively.

\section{Statistical analysis}

Data were compared with two-tailed, paired Student's $t$-test or analysed using regression analysis (Pearson's). A $P$-value of $<0.05$ was considered statistically significant in all cases with data expressed as mean \pm SEM. The statistical analysis was performed using GraphPad Prism v5.0 software.

\section{Results}

Effect of aspirin on platelet eicosanoid levels

One of the main eicosanoids detected from collagenstimulated platelets was $\mathrm{TxB}_{2}$, the stable isomer of $\mathrm{TxA}_{2}$ and a surrogate measure of $\mathrm{TxA}_{2}$ generation. Prior to aspirin treatment there was a wide distribution of $\mathrm{TxB}_{2}$ concentrations in the study cohort with a mean value of $13.8 \pm 1.6 \mathrm{ng} / 75 \times 10^{6}$ platelets (mean $\left.\pm \mathrm{SEM}\right)$. In keeping with the recognised concept of aspirin activity as a COX-1 inhibitor, oral administration of low-dose aspirin led to a statistically significant decrease in platelet $\mathrm{TxB}_{2}$ concentrations in all of the subjects, resulting in a mean $\mathrm{TxB}_{2}$ concentration of $1.7 \pm 0.3 \mathrm{ng} / 75 \times 10^{6}$ platelets (Figure 1A).

Interestingly, aspirin also induced a highly statistically significant $(P<0.0001)$ decrease in platelet 12 -HETE generation, reducing levels prior to stimulation from a mean of $6.2 \mathrm{ng}$ to $2.9 \pm 0.7 \mathrm{ng} / 75 \times 10^{6}$ platelets (Figure 1B). This suggests that, in addition to inhibition of COX-1, aspirin also impacts upon the 12-LOX pathway to decrease 12-HETE levels. In contrast to $\mathrm{TxB}_{2}$, 12-HETE inhibition was less pronounced and more variable among subjects (mean inhibition $=53 \pm 20 \%$; range, $20-84 \%$ ), whereas $\mathrm{TxB}_{2}$ inhibition was strong and consistent between subjects (mean inhibition $=88 \pm 7 \%$; range, 78-95\%). Whilst considerable inter-subject variation in both $\mathrm{TxB}_{2}$ and 12-HETE levels pre-aspirin treatment was observed, when the relative amounts of $\mathrm{TxB}_{2}$ and 12-HETE were compared, the 12-HETE:TxB molar ratio was found to be similar in all subjects, regardless of the initial eicosanoid concentration (Figure 1C). However, post-aspirin treatment, this ratio was considerably higher, a result that indicates an excess of 12-HETE compared to $\mathrm{TxB}_{2}$ in the platelets. Concentrations of $\mathrm{PGE}_{2}$ were lower
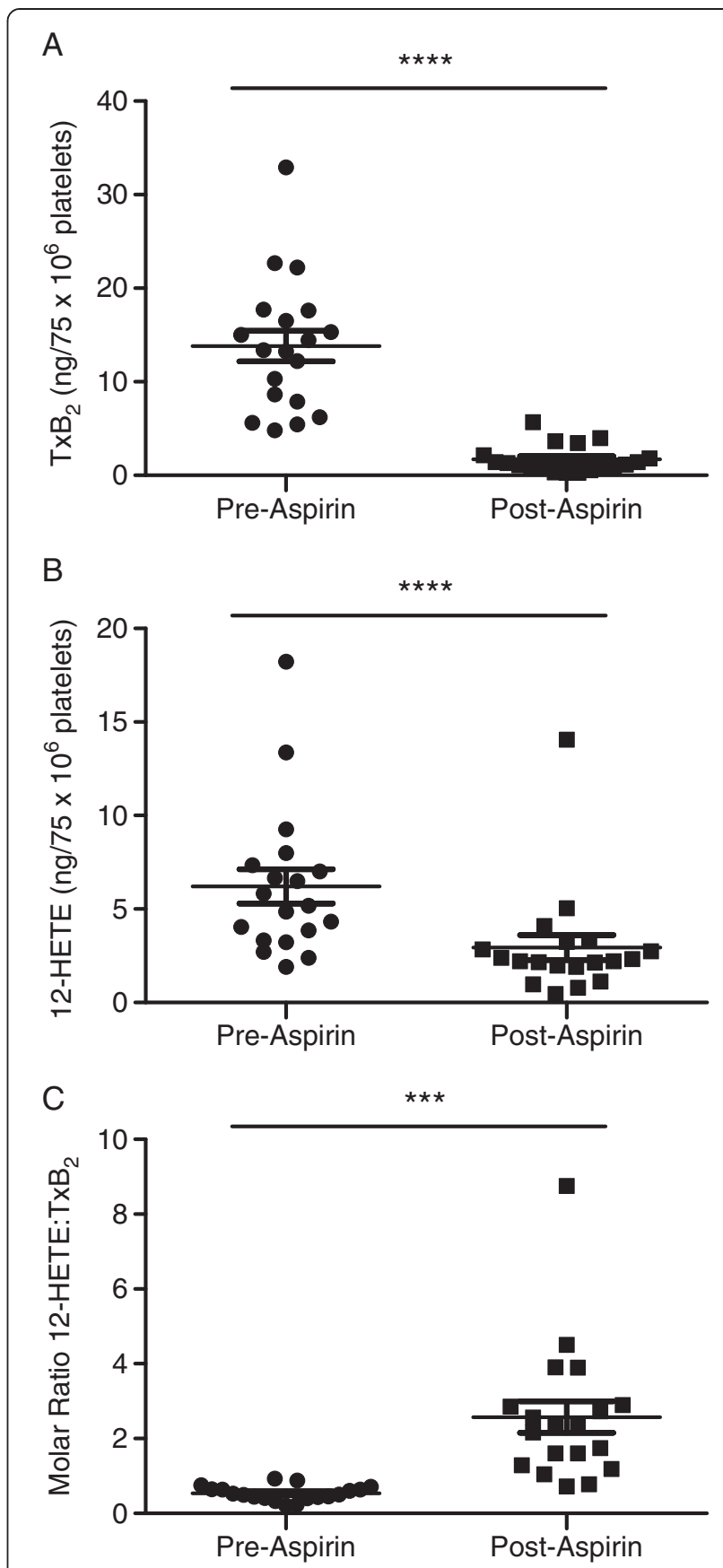

Figure 1 Low-dose aspirin decreases levels of $\mathrm{TxB}_{2}$ and 12-HETE. Concentrations of (A) $\mathrm{TxB}_{2}$ and (B) 12-HETE were reduced in collagen-activated platelets following $75 \mathrm{mg}$ aspirin oral administration to healthy volunteers $(n=19)$. (C) 12-HETE:TXB $B_{2}$ molar ratio is elevated following aspirin-treatment. Data are expressed as mean $\pm \mathrm{SEM}$. ${ }^{* * * *} P<0.0001$ and ${ }^{* * *} P<0.0005$.

than $\mathrm{TxB}_{2}$ and 12-HETE, with a mean value of $0.46 \pm$ $0.06 \mathrm{ng} / 75 \times 10^{6}$ platelets. Following aspirin administration $\mathrm{PGE}_{2}$ was undetectable in the majority of samples (data not shown). 


\section{Aspirin and platelet aggregation}

Considering the functional response of platelets to aspirin treatment, low-dose aspirin treatment significantly $(P<0.0001)$ decreased the platelet aggregatory response to collagen in all subjects (Figure 2). The greatest observed aspirin-mediated reduction in platelet aggregation was $97 \%$, whilst the lowest reduction in aggregation caused by aspirin resulted in only a $47 \%$ decrease in the aggregatory response.

\section{Correlations between eicosanoid levels and platelet aggregation}

When eicosanoid levels generated from platelets postaspirin treatment were compared with the aspirin-induced reduction in aggregation, a statistically significant correlation $(P=0.02$; Pearson correlation coefficient of $r=0.52)$ was observed between $\mathrm{TxB}_{2}$ levels and aspirin-mediated decrease in platelet aggregation (Figure 3A). Elevated $\mathrm{TxB}_{2}$ levels were associated with smaller reductions in aggregation, whilst the lowest levels of $\mathrm{TxB}_{2}$ correlated with the greatest percentage decrease in aggregation. However, as shown in Figure 3B, an even stronger relationship was found to exist between post-aspirin 12-HETE levels and the platelet aggregatory response, which was statistically significant $(P=0.0003)$ and produced a Pearson correlation coefficient of $r=0.74$. Similar to $\mathrm{TxB}_{2}$, the lowest levels of 12-HETE correlated with the greatest aspirininduced reduction in aggregation.

\section{2-LOX inhibition and platelet aggregation}

To investigate a functional role for 12-HETE in platelet aggregation, 12-LOX was inhibited using baicalein. As shown in Figure 4A, treatment with $100 \mu \mathrm{M}$ baicalein significantly reduced $(P=0.032)$ platelet aggregation in response to collagen activation (mean reduction in

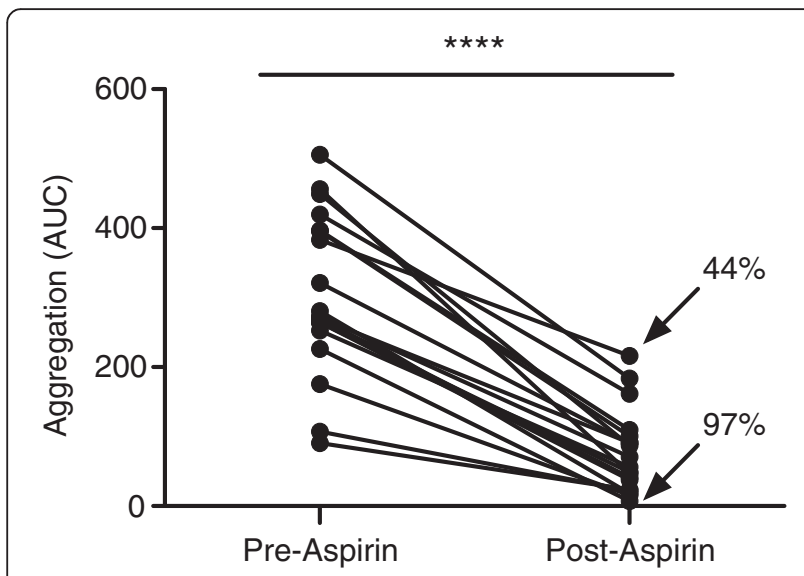

Figure 2 Platelet aggregation is decreased by low-dose aspirin. Collagen-induced platelet aggregation is significantly decreased following oral administration of $75 \mathrm{mg}$ aspirin to healthy volunteers $(n=19)$. Data are expressed as mean \pm SEM. ${ }^{* * *} P<0.0001$.

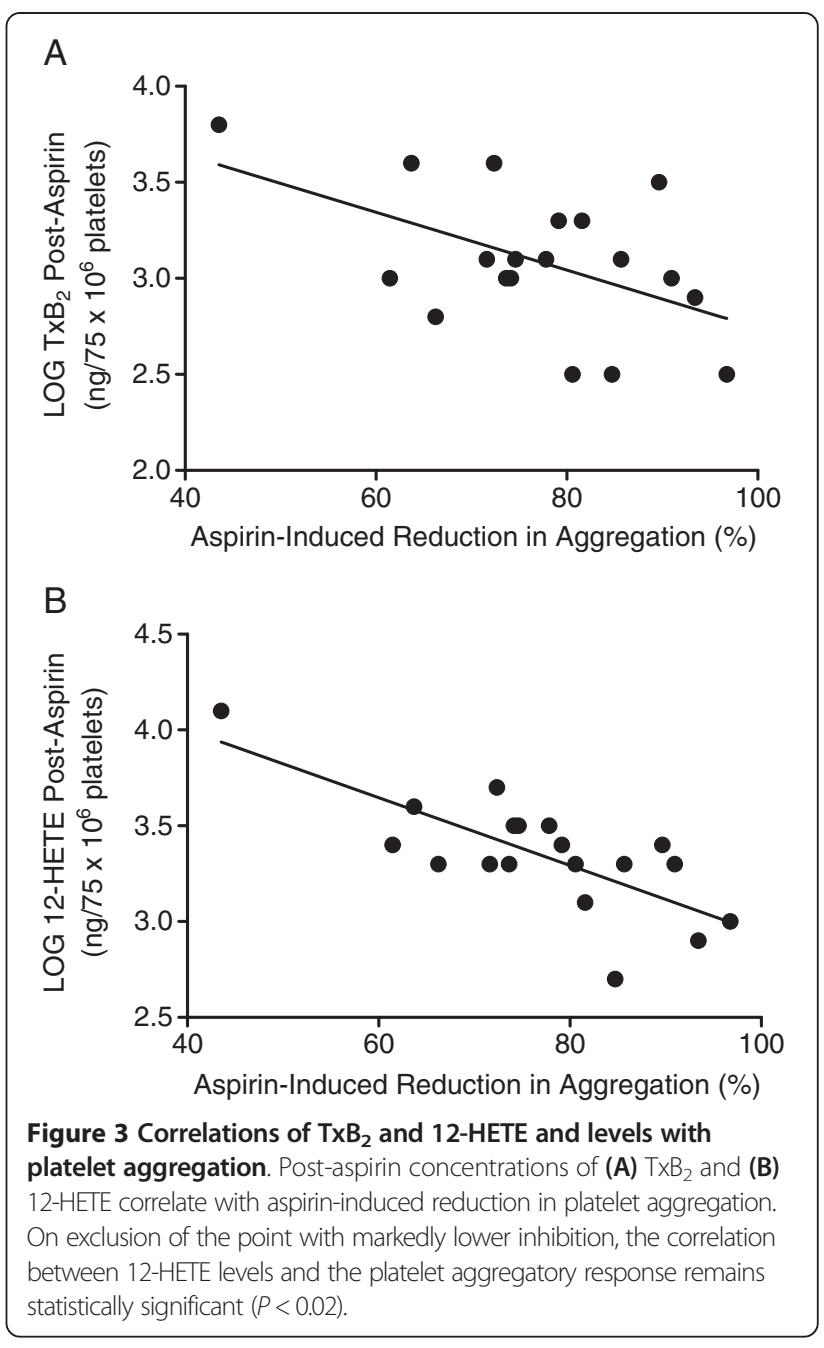

aggregation $=55 \pm 11 \%$ ). Figure $4 \mathrm{~B}$ shows representative aggregometry traces. To confirm effective baicaleininduced inhibition of 12-LOX, levels of 12-HETE were measured by LC-MS/MS and revealed a mean reduction in 12 -HETE to $52 \pm 6 \%$ with baicalein treatment.

\section{Discussion}

These findings demonstrate that, as well as inhibiting $\mathrm{TxA}_{2}$ generation through $\mathrm{COX}-1$, aspirin additionally impacts upon 12-LOX-mediated synthesis of 12-HETE. Whilst the effect of aspirin on $\mathrm{TxA}_{2}$ was universal and comprehensive, indicating effective aspirin-mediated COX-1 inhibition, the variation in efficacy on 12-HETE inhibition was also closely associated with the extent of inhibition of aggregation. Indeed, the subject with the highest levels of 12-HETE was found to have the lowest aspirin-induced reduction in platelet aggregation. This suggests that aspirin-mediated inhibition of 12-HETE generation may be of use in predicting the efficacy of aspirin with regard to platelet function. Whilst generally considered to be a classic COX-1 inhibitor, aspirin has 


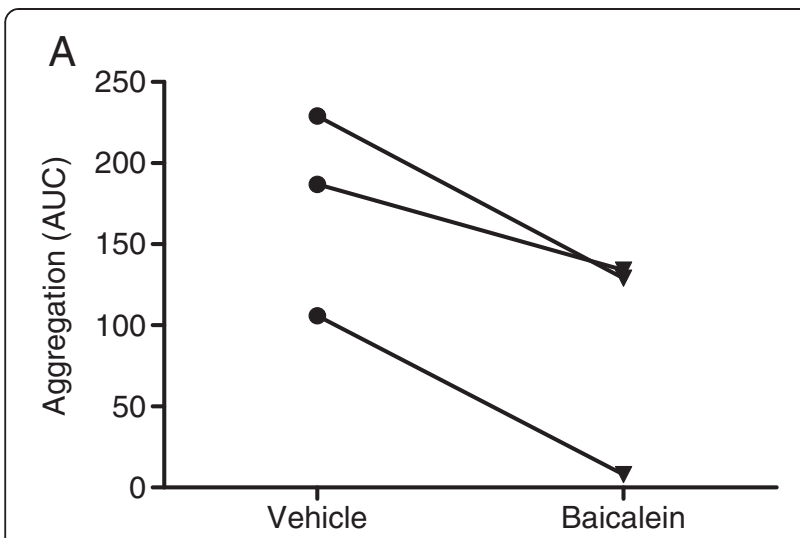

B

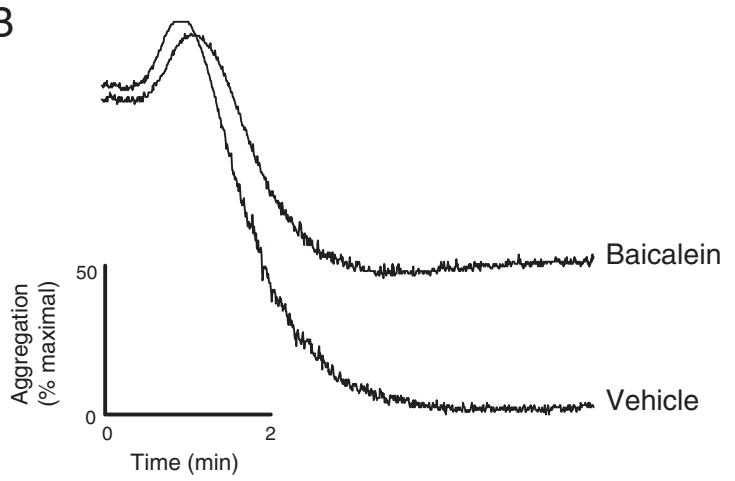

Figure 4 Inhibition of 12-LOX reduces platelet aggregation.

Platelets were pre-treated with or without $100 \mu \mathrm{M}$ baicalein prior to aggregation with $1.25 \mathrm{\mu g} / \mathrm{ml}$ collagen. Platelet aggregation was measured for 6 min and data expressed as (A) area under the curve (AUC). $(n=3$, mean \pm SEM). Panel B shows a representative aggregometry trace.

previously been shown to impact upon the platelet 12 LOX pathway, and can reversibly inhibit 12-HETE generation in a dose-dependent manner $[17,18]$. This effect appears to be unique to aspirin rather than being due to secondary effects caused by COX inhibition as treatment with indomethacin (COX-1 inhibitor) has no impact upon 12-HETE levels [19].

Considerable heterogeneity of platelet response to aspirin was observed between the healthy volunteers in this study, despite effective $\mathrm{TxB}_{2}$ inhibition. This observation parallels a recent study which showed that aspirin reduced $\mathrm{TxB}_{2}$ levels in all subjects tested [20] further supporting the notion that an alternative mechanism to $\mathrm{TxB}_{2}$ may be involved in variable sensitivity to aspirin. Our study examined the acute (1.5 hours) effect of aspirin treatment on eicosanoid levels and platelet function. Despite aspirin being an irreversible inhibitor of platelet COX-1, it is recognised that cumulative dosing of aspirin may be required to achieve maximal inhibition of $\mathrm{TxB}_{2}$ generation, although how cumulative dosing effects 12-HETE inhibition are currently unknown [21].
12-HETE has been suggested to play a role in platelet function and is thought to contribute to both platelet adhesion and aggregation $[19,22]$ although its exact role is unclear. Addition of 12-HpETE, the hydroperoxy precursor of 12-HETE has been demonstrated to play a 'priming' role and increase aggregation of washed platelets [23,24]. However, other studies have shown that addition of exogenous 12-HpETE inhibits both collagen and thrombininduced platelet aggregation $[25,26]$ suggestive of antiaggregatory properties.

Pharmacological inhibition of 12-LOX diminishes aggregation of washed platelets, further suggesting a role for 12-HETE in promoting platelet aggregation [11,27,28]. Other evidence for a pro-aggregatory role of 12-HETE comes from a recent study that showed a significant decrease in aggregation of washed platelets in response to collagen with a newly developed inhibitor, NCTT-956 that is highly specific for 12-LOX [29]. These results are consistent with our experimental findings using baicalein, although it should be noted that this inhibitor may have off-target effects on other eicosanoid pathways. The functional importance of platelet 12-LOX is also emphasized by the fact that 12-LOX-deficient mice display elevated mortality in an ADP-induced model of thromboembolism [30] and inhibition of 12-LOX inhibit coronary thrombosis in a canine model [31]. Furthermore, in patients receiving heparin treatment, platelet-derived 12-HETE has been shown to be elevated, whilst $\mathrm{TxB}_{2}$ levels were unchanged [32]. This was suggested as a possible mechanism to explain the phenomenon of transient heparin-induced platelet activation.

The signalling pathways involved in eicosanoid-dependent platelet activation are complex and not yet fully understood. Collagen is known to stimulate platelet 12-LOX activity via the glycoprotein VI receptor, in a PECAM-1 and PKCdependent pathway [33]. Release of AA from membrane phospholipids by $\mathrm{PLA}_{2}$ activation is a critical step in eicosanoid biosynthesis and a recent study has shown that COX-1 and 12-LOX are linked to different $\mathrm{PLA}_{2}$ subsets, suggesting that $\mathrm{TxA}_{2}$ and 12-HETE are generated from different intracellular pools of AA [34]. 12-HpETE is known to modulate platelet COX activity, suggesting a degree of 'cross-talk' between the LOX/COX pathways although the exact mechanism is unclear.

Some studies have reported a stimulatory effect of low concentrations of 12-HpETE on COX-1 activity [30], whilst others have shown that 12-HpETE inhibits COX-1 by altering calcium homeostasis via activation of soluble guanylate cyclase [26]. The exact nature of the inhibitory mechanism of aspirin on the 12-LOX pathway is unclear at present. However, it has been suggested that aspirin exerts its impact by inhibiting the glutathione peroxidase-mediated conversion of 12-HpETE to 12-HETE, preventing 12-HETE formation [35]. COX-1 has a bifunctional activity producing 
the labile peroxide, $\mathrm{PGG}_{2}$, which is reduced to the alcohol $\mathrm{PGH}_{2}$ via an endoperoxidase mechanism. $\mathrm{PGH}_{2}$ is then further metabolised by various prostanoid-generating enzymes. This bears a striking similarity to the mechanism of 12-HETE generation, whereby 12-LOX catalyses the regioand stereo-specific incorporation of molecular oxygen to form the hydroperoxide (12-HpETE), which is then reduced to the corresponding alcohol (12-HETE). Therefore, it is possible that aspirin may be acting in a similar manner, and preventing the reduction of 12 -HpETE.

Aspirin remains, and is likely to remain for some time, the mainstay of anti-platelet therapy in vascular patients and so a full understanding of its mechanisms of action is essential. Here we have shown for the first time a correlation between aspirin-mediated platelet aggregation and 12-HETE generation. This finding highlights the importance of the platelet 12-LOX pathway as an additional mechanism by which aspirin can modulate the complex network of AA metabolism, with potential effects on thrombotic events. This study also underlines the importance of monitoring interconnecting pathways when investigating the inhibitory mechanism of pharmacological agents. In future work the investigation of the mechanism and nature of aspirin inhibition of 12-LOX could be extended by using platelet markers of activation ex vivo e.g. P-selectin, larger sample sets and disease cohorts such as patients with type 2 diabetes or cardiovascular disease.

\section{Conclusions}

This study draws attention to the potential importance of the 12-LOX pathway in platelet function, specifically aspirin-mediated platelet inhibition. The findings suggest that the variability of platelet function in response to aspirin is linked to the inhibition of 12-HETE generation, which may be acting in concert with $\mathrm{TxA}_{2}$ inhibition. As such, the work may have implications for our understanding into how aspirin impacts upon eicosanoid generation.

\section{Abbreviations}

AA: Arachidonic acid; COX: Cyclooxygenase; HETE: Hydroxyeicosatetraenoic acid; HpETE: Hydroperoxyeicosatetraenoic acid; LOX: Lipoxygenase; NSAID: Nonsteroidal anti-inflammatory drug; PG: Prostaglandin; PRP: Platelet-rich plasma; Tx: Thromboxane.

\section{Competing interests}

The authors declare that they have no competing interests.

\section{Authors' contributions}

$\mathrm{BHM}$ performed the mass spectrometric analysis of eicosanoids. BHM, MHL and ATT carried out the platelet aggregation studies. BHM, GFR, JW, SJL, ILM and PDW conceived the study, participated in its design, contributed to the interpretation of the results and helped to draft the manuscript. All authors read and approved the final manuscript.

\section{Acknowledgments}

The financial support of the European Regional Development Fund, Scottish Funding Council, Highlands and Islands Enterprise and NHS Highland Research and Development Endowment Fund is gratefully acknowledged.

\section{Author details}

'Department of Diabetes and Cardiovascular Science, University of the Highlands and Islands, Old Perth Road, Inverness IV2 3JH, UK. ${ }^{2}$ Highland Clinical Research Facility, Inverness, UK. ${ }^{3}$ Cardiac Unit, Raigmore Hospital, Inverness, UK.

Received: 19 August 2014 Accepted: 8 October 2014

Published online: 23 October 2014

\section{References}

1. Cronstein BN, Weissmann G: Targets for antiinflammatory drugs. Annu Rev Pharmacol Toxicol 1995, 35:449-462.

2. Antithrombotic Trialists' Collaboration: Collaborative meta-analysis of randomised trials of antiplatelet therapy for prevention of death, myocardial infarction, and stroke in high risk patients. BMJ 2002, 324:71-86.

3. Vane JR: Inhibition of prostaglandin synthesis as a mechanism of action for aspirin-like drugs. Nat New Biol 1971, 231:232-235.

4. Roth GJ, Stanford N, Majerus PW: Acetylation of prostaglandin synthase by aspirin. Proc Natl Acad Sci U S A 1975, 72:3073-3076.

5. Roth GJ, Calverley DC: Aspirin, platelets, and thrombosis: theory and practice. Blood 1994, 83:885-898.

6. Hankey GJ, Eikelboom JW: Aspirin resistance. Lancet 2006, 367:606-617.

7. Mason PJ, Jacobs AK, Freedman JE: Aspirin resistance and atherothrombotic disease. J Am Coll Cardio/ 2005, 46:986-993.

8. Krasopoulos G, Brister SJ, Beattie WS, Buchanan MR: Aspirin "resistance" and risk of cardiovascular morbidity: systematic review and metaanalysis. BMJ 2008, 336:195-198.

9. Santilli F, Rocca B, De Cristofaro R, Lattanzio S, Pietrangelo L, Habib A, Pettinella C, Recchiuti A, Ferrante E, Ciabattoni G, Davi G, Patrono C: Platelet cyclooxygenase inhibition by low-dose aspirin is not reflected consistently by platelet function assays: implications for aspirin "resistance". J Am Coll Cardiol 2009, 53:667-677.

10. Takenaga M, Hirai A, Terano T, Tamura Y, Kitagawa H, Yoshida S: Comparison of the in vitro effect of eicosapentaenoic acid (EPA)-derived lipoxygenase metabolites on human platelet function with those of arachidonic acid. Thromb Res 1986, 41:373-384.

11. Nyby MD, Sasaki M, Ideguchi Y, Wynne HE, Hori MT, Berger ME, Golub MS, Brickman AS, Tuck ML: Platelet lipoxygenase inhibitors attenuate thrombin- and thromboxane mimetic-induced intracellular calcium mobilization and platelet aggregation. J Pharmacol Exp Ther 1996, 278:503-509.

12. Sekiya F, Takagi J, Sasaki K, Kawajiri K, Kobayashi Y, Sato F, Saito Y: Feedback regulation of platelet function by $12 \mathrm{~S}$-hydroxyeicosatetraenoic acid: inhibition of arachidonic acid liberation from phospholipids. Biochim Biophys Acta 1990, 1044:165-168.

13. Ozeki $Y$, Ito H, Nagamura $Y$, Unemi F, Igawa T: 12(S)-HETE plays a role as a mediator of expression of platelet CD62 (P-selectin). Platelets 1998, 9:297-302.

14. González-Núñez D, Claria J, Rivera F, Poch E: Increased levels of 12(S)-HETE in patients with essential hypertension. Hypertension 2001, 37:334-338.

15. Kenyon V, Rai G, Jadhav A, Schultz L, Armstrong M, Jameson JB, Perry S, Joshi N, Bougie JM, Leister W, Taylor-Fishwick DA, Nadler JL, Holinstat M, Simeonov A, Maloney DJ, Holman TR: Discovery of potent and selective inhibitors of human platelet-type 12- lipoxygenase. J Med Chem 2011, 54:5485-5497.

16. Yeung J, Holinstat M: 12-lipoxygenase: a potential target for novel antiplatelet therapeutics. Cardiovasc Hematol Agents Med Chem 2011, 9:154-164.

17. Eynard AR, Galli G, Tremoli E, Maderna P, Magni F, Paoletti R: Aspirin inhibits platelet 12-hydroxy-eicosatetraenoic acid formation. J Lab Clin Med 1986, 107:73-78.

18. Tremoli E, Maderna P, Eynard A, Gregori M, Galli G: In vitro effects of aspirin and non steroidal anti-inflammatory drugs on the formation of 12-hydroxyeicosatetraenoic acid by platelets. Prostaglandins Leukot Med 1986, 23:117-122.

19. Maderna P, Caruso D, Tremoli E, Galli G: Differential effects of oral administrations to human volunteers of acetylsalicylic acid, sodium salicylate and indomethacin on 12-hydroxyeicosatetraenoic acid formation by stimulated platelets. Thromb Res 1988, 52:197-206.

20. Grosser T, Fries S, Lawson JA, Kapoor SC, Grant GR, FitzGerald GA: Drug resistance and pseudoresistance: an unintended consequence of enteric coating aspirin. Circulation 2013, 127:377-385. 
21. Patrignani $P$, Filabozzi $P$, Patrono C: Selective cumulative inhibition of platelet thromboxane production by low-dose aspirin in healthy subjects. J Clin Invest 1982, 69:1366-1372.

22. Buchanan MR, Butt RW, Hirsh J, Markham BA, Nazir DJ: Role of lipoxygenase metabolism in platelet function: effect of aspirin and salicylate. Prostaglandins Leukot Med 1986, 21:157-168.

23. Calzada C, Vericel E, Lagarde M: Low concentrations of lipid hydroperoxides prime human platelet aggregation specifically via cyclo-oxygenase activation. Biochem J 1997, 325:495-500.

24. Calzada C, Véricel E, Mitel B, Coulon L, Lagarde M: 12(S)-Hydroperoxyeicosatetraenoic acid increases arachidonic acid availability in collagen-primed platelets. J Lipid Res 2001, 42:1467-1473.

25. Aharony D, Smith JB, Silver MJ: Regulation of arachidonate-induced platelet aggregation by the lipoxygenase product, 12-hydroperoxyeicosatetraenoic acid. Biochim Biophys Acta 1982, 718:193-200.

26. Brüne B, Ullrich V: 12-hydroperoxyeicosatetraenoic acid inhibits main platelet functions by activation of soluble guanylate cyclase. $\mathrm{Mol}$ Pharmacol 1991, 39:671-678.

27. Yeung J, Apopa PL, Vesci J, Stolla M, Rai G, Simeonov A, Jadhav A, Fernandez-Perez P, Maloney DJ, Boutaud O, Holman TR, Holinstat M: 12-lipoxygenase activity plays an important role in PAR4 and GPVI-mediated platelet reactivity. Thromb Haemost 2013, 110:569-581.

28. Huang W-H, Lee A-R, Chien P-Y, Chou T-C: Synthesis of baicalein derivatives as potential anti-aggregatory and anti-inflammatory agents. J Pharm Pharmacol 2005, 57:219-225.

29. Yeung J, Apopa PL, Vesci J, Kenyon V, Rai G, Jadhav A, Simeonov A, Holman TR, Maloney DJ, Boutaud O, Holinstat M: Protein kinase C regulation of 12lipoxygenase-mediated human platelet activation. Mol Pharmacol 2012. 81:420-430.

30. Johnson EN, Brass LF, Funk CD: Increased platelet sensitivity to ADP in mice lacking platelet-type 12-lipoxygenase. Proc Natl Acad Sci U S A 1998, 95:3100-3105.

31. Katoh A, Ikeda H, Murohara T, Haramaki N, Ito H, Imaizumi T: Platelet-derived 12-hydroxyeicosatetraenoic acid plays an important role in mediating canine coronary thrombosis by regulating platelet glycoprotein $\mathrm{llb} / \mathrm{llla}$ activation. Circulation 1998, 98:2891-2898.

32. McMahon GS, Jones Cl, Hayes PD, Naylor AR, Goodall AH: Transient heparin-induced platelet activation linked to generation of platelet 12lipoxygenase. Findings from a randomised controlled trial. Thromb Haemost 2013, 109:1099-1107.

33. Coffey MJ, Jarvis GE, Gibbins JM, Coles B, Barrett NE, Wylie ORE, O'Donnell VB: Platelet 12-lipoxygenase activation via glycoprotein VI: involvement of multiple signaling pathways in agonist control of H(P)ETE synthesis. Circ Res 2004, 94:1598-1605.

34. Holinstat M, Boutaud O, Apopa PL, Vesci J, Bala M, Oates JA, Hamm HE: Protease-activated receptor signaling in platelets activates cytosolic phospholipase A2a differently for cyclooxygenase-1 and 12lipoxygenase catalysis. Arterioscler Thromb Vasc Biol 2011, 31:435-442.

35. Siegel MI, McConnell RT, Cuatrecasas P: Aspirin-like drugs interfere with arachidonate metabolism by inhibition of the 12-hydroperoxy-5,8,10,14eicosatetraenoic acid peroxidase activity of the lipoxygenase pathway. Proc Natl Acad Sci U S A 1979, 76:3774-3778

\section{doi:10.1186/s12950-014-0033-4}

Cite this article as: Maskrey et al.: 12-hydroxyeicosatetraenoic acid is associated with variability in aspirin-induced platelet inhibition. Journal of Inflammation 2014 11:33.

\section{Submit your next manuscript to BioMed Central and take full advantage of:}

- Convenient online submission

- Thorough peer review

- No space constraints or color figure charges

- Immediate publication on acceptance

- Inclusion in PubMed, CAS, Scopus and Google Scholar

- Research which is freely available for redistribution 